\title{
The elastic-viscous-plastic method revisited
}

\author{
Sylvain Bouillon ${ }^{\mathrm{a}, *}$, Thierry Fichefet ${ }^{\mathrm{b}}$, Vincent Legat ${ }^{\mathrm{b}, \mathrm{c}}$, Gurvan Madec ${ }^{\mathrm{d}, \mathrm{e}}$ \\ ${ }^{a}$ Nansen Environmental and Remote Sensing Center (NERSC), Bergen, Norway \\ ${ }^{\mathrm{b}}$ Georges Lemaître Centre for Earth and Climate Research, Earth and Life Institute, Université catholique de Louvain, Louvain-la-Neuve, Belgium \\ ${ }^{\mathrm{c}}$ Institute of Mechanics, Materials and Civil Engineering, Université catholique de Louvain, Louvain-la-Neuve, Belgium \\ d Laboratoire d'Océanographie et du Climat: Expérimentation et Approches Numériques (LOCEAN), Paris, France

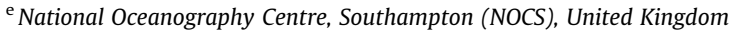

\section{A R T I C L E I N F O}

\section{Article history:}

Available online 17 June 2013

\section{Keywords:}

Viscous-plastic rheology

Elastic-viscous-plastic

Sea ice

NEMO

\begin{abstract}
A B S T R A C T
In this paper, we show that one of the most widely used methods to solve the non-linear viscous-plastic (VP) sea ice rheology, the elastic-viscous-plastic (EVP) method, generates artificial linear bands of high deformation that may be confounded with real linear kinematic features observed in the Arctic ice pack. These numerical artefacts are easily filtered out by using a slightly different regularization of the internal stress. In addition, the EVP method is reinterpreted as an iterative solver and a clear distinction appears between the numerical and physical parameters. Two numerical parameters determine the stability and accuracy of the method and are adjusted to avoid the noisy ice deformation fields frequently observed with the EVP method in nearly rigid ice areas. This study also confirms the unsatisfactory numerical convergence of the EVP method and investigates the effects of the numerical parameters on sea ice deformation, internal stress and velocity fields obtained with unconverged solutions.
\end{abstract}

(c) 2013 Elsevier Ltd. All rights reserved.

\section{Introduction}

Most large-scale sea ice models use some variants of the viscous-plastic (VP) rheology (Hibler, 1979) and produce realistic mean ice drift patterns (Martin and Gerdes, 2007). However, there is evidence that numerical sea ice models do not reproduce ice deformation fields at small spatial and temporal scales (Kwok et al., 2008; Girard et al., 2009). This raises questions about the principal assumptions on which plastic sea ice rheologies rely (Coon et al., 2007) and generates renewed interest in setting up new frameworks for sea ice dynamics (e.g., Hibler and Schulson, 2000; Wilchinsky and Feltham, 2004; Schreyer et al., 2006; Girard et al., 2011). On the other hand, some authors claim that higher resolution combined with appropriate plastic yield curves allows the VP rheology to simulate bands of high ice deformation (e.g., Wang and Wang, 2009; Maslowski and Lipscomb, 2003) similar to the linear kinematic features observed in the Arctic (Kwok, 2006). This paper participates in this discussion by showing that the generation of some linear kinematic features by sea ice models are due to numerical artefacts.

One of the most commonly used method to find numerical solutions for ice trajectories is the elastic-viscous-plastic (EVP) method which is thoroughly documented in Hunke and Dukowicz (1997), Hunke and Dukowicz (2002) and Hunke and Lipscomb,

\footnotetext{
* Corresponding author. Tel.: +4755205800.

E-mail address: sylvain.bouillon@nersc.no (S. Bouillon).
}

2008. We will refer here to the classical version presented in Hunke (2001) and to the modified version proposed by Lemieux et al. (2012). The EVP method is based on subcycling iterations during which the ice velocities and internal stresses are successively updated towards the solution of the non-linear VP equations. Relaxation terms, also called regularization terms, are introduced to avoid instabilities. In the classical version, the regularization consists in adding an artificial elastic term whereas the modified version also relies on an extra inertial term to control the relaxation to the solution of the VP equations. These terms may generate oscillations of the solution which need to be damped before the end of the iterative process.

The trivial parallelization of the EVP method explains its success in coupled ice-ocean models. However, this method presents several drawbacks. Several studies showed that the EVP method may not convergence (or very slowly) to the solution of the VP equation (Losch and Danilov, 2012; Lemieux et al., 2012). The insufficient numerical convergence implies a strong impact of the numerical parameters on the results (Losch et al., 2010). Another serious problem, coming from numerical instabilities or a lack of damping, is the generation of noisy deformation fields in nearly rigid ice areas (Hunke, 2001), leading to large errors in the simulated thickness and concentration fields (Lemieux et al., 2012). This problem is critical in the Canadian Arctic Archipelago (CAA) where deformation rates are very low and ice thickness is relatively high.

Other methods are also used to solve the non-linear VP equations. Most of them are based on the linearization of the equations obtained from the implicit time discretization of the VP model. The 
resulting system of equations is solved with various linear solvers. As the non-linear terms are linearized by using the velocities from the previous timestep, the solution is supposed to be correctly approximated if the timestep is much smaller than the forcing timescale. This constraint is relaxed by using outer-loop iterations (sometimes referred to as Picard iterations or pseudo time steps) during which forcing is held constant (Zhang and Hibler, 1997; Zhang and Rothrock, 2000) and the non-linear terms are linearized with the velocities from the previous iteration. In all cases, a linear solver is needed, making the parallelization of the code more complex. These methods usually converge but with poor rates of convergence (Lemieux and Tremblay, 2009).

An interesting approach is the use of general non-linear solvers. Lemieux et al. (2010) implemented a Jacobian-free Newton-Krylov method which is based on a more sophisticated linearization of the VP equations. The resulting matrix also contains derivatives relative to the unknowns, speeding up the convergence of the non-linear problem. A linear solver is still needed and the efficiency has not been evaluated in parallel environments.

The first goal of our study was the mitigation of the noise in the deformation fields produced by the EVP method in nearly rigid ice regions. By reinterpreting EVP as an iterative solver, we define clear criteria for stability and damping, leading to a better control of the noise in the deformation fields. This reinterpretation of the EVP method also leads to a new regularization of the internal stress ensuring the same relaxation for all the stress components. With the old regularization (initially proposed by Hunke and Dukowicz (1997) and used in nearly all EVP implementations), results may exhibit linear bands of high ice deformation aligned with the grid. These bands are not obtained with the new regularization which has also lower remaining errors.

This paper is organized in three sections. Section 2 briefly presents the VP equations. Section 3 presents their spatial and temporal discretizations, along with the reinterpretation of EVP and the new regularization of the internal stress. The stability and damping criteria are analytically derived from a simplified one-dimensional problem. Several simulations are presented in Section 4 to illustrate the effects of numerical parameters on the behaviour of the method and on the quality of the solutions obtained with a limited number of iterations. A comparison of results obtained with both the old and new regularizations of the internal stress shows the strong impact of numerical details on the generation of linear bands of high ice deformation, and their correlation with large remaining errors in the velocity field.

\section{Model description}

The viscous-plastic sea ice rheology proposed by Hibler (1979) is widely used in many large-scale ice-ocean models (e.g., CCSM, HadGEM3, MITgcm and NEMO-LIM). It is based on the assumption that sea ice has no (or little) tensile strength but responds in a plastic way to compressive and shear deformations. Combined with a parameterization of the ice strength, this model is able to reproduce the mean ice drift reasonably well and leads to a realistic ice thickness distribution within the Arctic basin (e.g., Zhang and Rothrock, 2000).

The momentum equation obtained by integrating the threedimensional momentum equation through the snow and ice in the vertical direction is

$m \partial_{t} \boldsymbol{u}=\nabla \cdot \boldsymbol{\sigma}+A\left(\tau_{a}+\tau_{w}\right)-m f \boldsymbol{k} \times \boldsymbol{u}-m g \nabla \eta$,

where $m$ is the total mass of snow and ice per unit area, $\partial_{t}$ is the temporal derivative, $\boldsymbol{u}$ is the horizontal ice velocity and $\boldsymbol{\sigma}$ is the internal ice stress tensor. The momentum advection is ignored because inertial forces are small compared to other forces in most applications (Fichefet et al., 1998). The wind stress $\tau_{a}$ and the ocean stress $\tau_{w}$ are multiplied by the ice concentration, $A$, following the proper area scaling determined by Connolley et al. (2004) and Hunke and Dukowicz, 2003. $\tau_{a}$ is an external forcing and $\tau_{w}$ is given by a quadratic expression, $c_{D} \rho_{o}\left|\boldsymbol{u}_{0}-\boldsymbol{u}\right|\left(\boldsymbol{u}_{0}-\boldsymbol{u}\right)$, where $c_{D}$ is the water drag coefficient, $\rho_{0}$ is the reference density of seawater and $\boldsymbol{u}_{o}$ is the oceanic surface velocity. The last two terms of Eq. (1) are related to the Coriolis effect and to the slope of the sea surface; $f$ is the Coriolis parameter, $\boldsymbol{k}$ is an unit vector normal to the reference surface of the Earth, $g$ is gravity and $\eta$ is the sea surface elevation. Definitions and units of symbols used in the text are detailed in Table 1.

The VP constitutive law relates the components of the strain rate tensor:

$\dot{\epsilon}_{i j}=\frac{1}{2}\left(\frac{\partial u_{i}}{\partial x_{i}}+\frac{\partial u_{j}}{\partial x_{j}}\right)$,

to the components of the internal ice stress tensor by:

$\sigma_{i j}=\frac{P}{2\left(\Delta+\Delta_{\min }\right)}\left[\left(\dot{\epsilon}_{k k}-\Delta\right) \delta_{i j}+\frac{1}{e^{2}}\left(2 \dot{\epsilon}_{i j}-\dot{\epsilon}_{k k} \delta_{i j}\right)\right]$,

where $P$ is the ice strength, $\Delta$ is a particular measure of the deformation rate and $\Delta_{\min }$ is a parameter determining a smooth transition from pure viscous flow $\left(\Delta \ll \Delta_{\min }\right)$ to pure plastic flow $\left(\Delta \gg \Delta_{\min }\right) . \dot{\epsilon}_{k k}$ is the trace of the strain rate tensor, $\delta_{i j}$ is the Kronecker unit tensor and $e$ is a parameter giving the ratio between the maximum compressive stress and twice the maximum shear stress. $\Delta$ is given by

$\Delta=\sqrt{\dot{\epsilon}_{d}^{2}+\frac{1}{e^{2}} \dot{\epsilon}_{s}^{2}}$

where $\dot{\epsilon}_{d}$ is the divergence rate and $\dot{\epsilon}_{s}$ is the shear strain rate, respectively given by $\dot{\epsilon}_{d}=\left(\dot{\epsilon}_{11}+\dot{\epsilon}_{22}\right)$ and $\dot{\epsilon}_{s}=\sqrt{\left(\dot{\epsilon}_{11}-\dot{\epsilon}_{22}\right)^{2}+4 \dot{\epsilon}_{12}^{2}}$. Note that $P /\left(2\left(\Delta+\Delta_{\min }\right)\right)$ in Eq. (3) is an alternative to the non-linear bulk viscosity with capping proposed by Hibler (1979). This formulation of the VP rheology is differentiable, well defined, and gives no internal stress when $\Delta$ tends to zero.

In the pure plastic case $\left(\Delta \gg \Delta_{\min }\right)$, the compressive stress $\sigma_{d}=\left(\sigma_{11}+\sigma_{22}\right)$ and the shear stress $\sigma_{s}=\sqrt{\left(\sigma_{11}-\sigma_{22}\right)^{2}+4 \sigma_{12}^{2}}$ are linked by the quadratic relationship $\left(\sigma_{d}+P\right)^{2}+e^{2} \sigma_{s}^{2}=P^{2}$, which defines an elliptical yield curve translated along the compressive stress to be entirely in the compressive part $\left(\sigma_{d} \leqslant 0\right)$ of the stress space. The position on that ellipse does not depend on the magnitude of the deformation rate but only on the direction of the strain rate vector (defined by $\dot{\epsilon}_{d}$ and $\dot{\epsilon}_{s}$ ) which must be normal to the yield curve.

According to Hibler (1979), the ice strength $P$ is parameterized as

Table 1

Definition and units of the variables used in the text.

\begin{tabular}{lll}
\hline Symbol & Definition & Units \\
\hline$P$ & ice strength & $\mathrm{N} \mathrm{m}^{-1}$ \\
$m$ & mass of snow and ice per unit area & $\mathrm{kg} \mathrm{m}^{-2}$ \\
$h$ & mean ice thickness & $\mathrm{m}$ \\
$A$ & ice concentration & - \\
$\Delta$ & measure of the deformation rate & $\mathrm{s}^{-1}$ \\
$\mathbf{F}=\left(F_{\mathrm{u}}, F_{\mathrm{v}}\right)$ & stress divergence & $\mathrm{N} \mathrm{m}^{-2}$ \\
$\dot{\epsilon}_{i j}$ & component of the strain rate tensor & $\mathrm{s}^{-1}$ \\
$\sigma_{i j}$ & component of the stress tensor & $\mathrm{N} \mathrm{m}^{-1}$ \\
$\boldsymbol{u}=(u, v)$ & ice velocity & $\mathrm{m} \mathrm{s}^{-1}$ \\
$\tau_{w}, \tau_{a}$ & ocean and atmosphere stresses & $\mathrm{N} \mathrm{m}^{-2}$ \\
$\eta$ & sea surface elevation & $\mathrm{m}$ \\
\hline
\end{tabular}


$P=P^{*} h e^{-C(1-A)}$,

where $P^{*}$ and $C$ are empirical positive parameters. $h$ is the mean ice thickness, corresponding to the volume of ice per unit area. Other yield curves, flow rules and strength parameterizations have been proposed (e.g., Zhang and Rothrock, 2005), but this combination of the elliptical yield curve, normal flow rule and Hibler's parameterization for the ice strength is very popular.

\section{The revised elastic-viscous-plastic method}

The EVP method is based on subcycling iterations during which the ice velocities and internal stresses are successively updated towards the solution of the coupled Eqs. (1) and (3). Regularization terms are introduced to avoid large oscillations and instabilities during the iterative process. In this study, the regularization consists in adding the term $2 T \partial_{t e} \sigma_{i j}$ to the left-hand side of Eq. (3). $T$ is the elastic damping timescale and $\partial_{t e}$ is a pseudo time derivative that represents the evolution of the solution during the iterative process. This new regularization is slightly different from the regularization initially proposed by Hunke and Dukowicz (1997), which consists in adding the same regularization term but to the left-hand side of the following equation, which is equivalent to Eq. (3):

$e^{2} \sigma_{i j}+\left(\frac{1-e^{2}}{2}\right) \sigma_{k k} \delta_{i j}+\frac{\Delta P}{\left(\Delta+\Delta_{\min }\right)} \delta_{i j}=\frac{P}{\left(\Delta+\Delta_{\min }\right)} \dot{\epsilon}_{i j}$.

This presentation of the EVP method is potentially confusing and the mixing between the numerical and physical terms may be problematic for the choice of parameters and the interpretation of results produced by this method. We then propose to revise the EVP method to have a clear distinction between the numerical and physical parameters. In the following, we first present the spatial discretization. We then describe the temporal discretization with a simple one-dimensional example and we explain our reinterpretation of the EVP method. The stability and damping properties are then analytically derived. Finally, the entire algorithm is detailed.

\subsection{The spatial discretization}

The spatial discretization is based on the C-grid arrangement of variables (Fig. 1 ) with cells centred on scalar points ( $t$-points). Velocity points ( $u$ - and $v$-points) are defined at the centre of each edge of the cells. In order to minimize the number of averaging operations for the evaluation of the different derivatives, $\dot{\epsilon}_{1}$ and $\dot{\epsilon}_{2}$ are defined as the values of $\left(\dot{\epsilon}_{11}+\dot{\epsilon}_{22}\right)$ and $\left(\dot{\epsilon}_{11}-\dot{\epsilon}_{22}\right)$, respectively, at the centre of each cell (t-points), whereas $\dot{\epsilon}_{12}$ is computed on the corners of the cells ( $f$-points). $\sigma_{1}$ and $\sigma_{2}$ are then naturally defined on $t$-points as $\left(\sigma_{11}+\sigma_{22}\right)$ and $\left(\sigma_{11}-\sigma_{22}\right)$ respectively by

$\sigma_{1}=\frac{P}{\left(\Delta+\Delta_{\min }\right)}\left(\dot{\epsilon}_{1}-\Delta\right)$,

$\sigma_{2}=\frac{P}{\left(\Delta+\Delta_{\min }\right)} \frac{\dot{\epsilon}_{2}}{e^{2}}$.

Similarly $\sigma_{12}$ is naturally defined on $f$-points by

$\sigma_{12}=\left.\frac{P}{\left(\Delta+\Delta_{\min }\right)}\right|_{f} \frac{\dot{\epsilon}_{12}}{e^{2}}$.

In the above equations, $\Delta$ is given by

$\Delta=\sqrt{\dot{\epsilon}_{1}^{2}+\frac{1}{e^{2}}\left(\dot{\epsilon}_{2}^{2}+\left.4 \dot{\epsilon}_{12}^{2}\right|_{t}\right)}$

and the averaging operators used to compute $\dot{\epsilon}_{12}$ on $t$-points and $P /\left(\Delta+\Delta_{\min }\right)$ on $f$-points are defined as

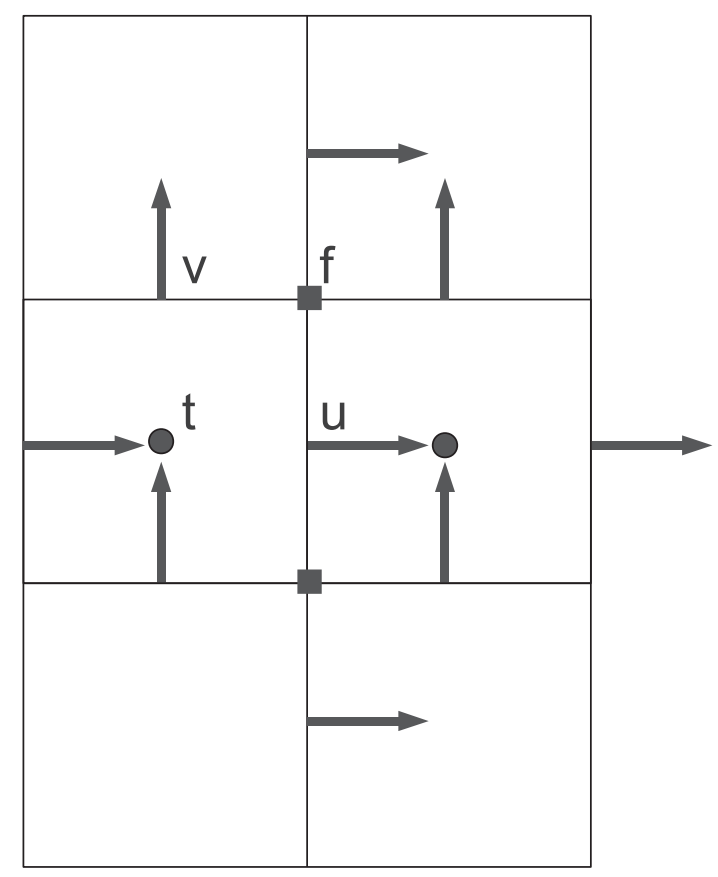

Fig. 1. Arrangement of variables on the C-grid. Velocities are defined on $u$ - and $v$ points. Scalars are defined on $t$-points, as well as $\sigma_{1}, \sigma_{2}, \dot{\epsilon}_{1}$ and $\dot{\epsilon}_{2} . \sigma_{12}$ and $2 \dot{\epsilon}_{12}$ are defined on $f$-points.

$\left.\dot{\epsilon}_{12}^{2}\right|_{t}=\frac{1}{e_{1 t} e_{2 t}} \overline{\overline{\dot{\epsilon}_{12}^{2} e_{1 f} e_{2 f}}} i, j$,

$\left.\frac{P}{\left(\Delta+\Delta_{\min }\right)}\right|_{f}={\frac{P}{\left(\Delta+\Delta_{\min }\right)}}_{i+\frac{1}{2}, j+\frac{1}{2}}$.

The quantities $e_{1 t}, e_{2 t}, e_{1 f}$ and $e_{2 f}$ are the grid lengths in each direction as defined at $\mathrm{t}$ - and $f$-points. The operator $\overline{\bar{q}}^{i, j}$ corresponds to

$\overline{\bar{q}}^{i, j}=\frac{q_{i, j}+q_{i, j-1}+q_{i-1, j}+q_{i-1, j-1}}{4}$,

where indices $i$ and $j$ cover all of the domain and indicate where a quantity is computed. $(i, j)$ corresponds to $t$-points, $\left(i+\frac{1}{2}, j\right)$ to $u$ points, $\left(i, j+\frac{1}{2}\right)$ to $v$-points and $\left(i+\frac{1}{2}, j+\frac{1}{2}\right)$ to $f$-points.

Apart from this averaging method, the spatial discretization is the same as that presented in Bouillon et al. (2009). This change ensures that the discretized equations satisfy the dissipative properties of the VP model but does not lead to noticeable changes in the results. The corrected discretization is used for all the experiments presented in this study.

\subsection{The temporal discretization}

In order to illustrate the temporal discretization and the reinterpretation of the EVP method, the following simplified onedimensional version of the VP problem is used:

$\sigma=\frac{P}{2 \Delta} \partial_{x} u$

$\Delta=\left|\partial_{x} u\right|$,

$m \partial_{t} u=\partial_{x} \sigma+\tau$

where $u$ is the ice velocity in the $x$ direction, $\tau$ is the external stress which is held constant and $\partial_{x}$ is the spatial derivative.

The simplest temporal discretization is an explicit scheme that advances from timestep $n$ to timestep $n+1$ using: 
$m \frac{u_{n+1}-u_{n}}{\Delta t}=\partial_{x} \frac{P}{2 \Delta_{n}} \partial_{x} u_{n}+\tau$

where $\Delta_{n}=\left|\partial_{x} u_{n}\right|$. The stability criterion scales quadratically with $\Delta x$ as

$\Delta t \leqslant \frac{m \Delta}{P} \Delta x^{2}$.

If the deformation rate $\Delta$ is $10^{-9} \mathrm{~s}^{-1}$, the spatial resolution $\Delta x=50 \mathrm{~km}, P=20000 \mathrm{~N} \mathrm{~m}^{-1}$ and $m=1000 \mathrm{~kg} \mathrm{~m}^{-2}$, the order of magnitude of the required timestep would be $0.1 \mathrm{~s}$. To allow larger timesteps, implicit schemes are required.

Solving the full implicit equations is not easy because of the presence of the term $P /(2 \Delta)$ in Eq. (14). First implementations simply used the value of $\Delta$ from the previous timestep by using $P /\left(2 \Delta_{n}\right)$ instead of solving the non-linear equations, but Hunke and Zhang (1999) showed that the resulting sea ice responds too slowly to forcing changes. An alternative is to solve the non-linear equation by using outer-loop iterations (Zhang and Hibler, 1997) where an additional loop of iterations is performed with indices $p$. The equations are linearized by using the velocity from the previous iteration.

$m \frac{u_{n+1}^{p+1}-u_{n}}{\Delta t}=\partial_{x} \frac{P}{2 \Delta_{n+1}^{p}} \partial_{x} u_{n+1}^{p+1}+\tau$.

The main drawback of this simple non-linear solver is the slow convergence towards the solution. Even with 40 outer-loop iterations, the velocity field can have significant errors (as shown by Lemieux and Tremblay (2009)). A solver (e.g., GMRES or LSOR) is called at each iteration to solve the linear system of equations. To speed up the convergence, Lemieux et al. (2010) proposed to use a Jacobian-free Newton-Krylov method to solve the non-linear set of equations but this method is not yet used in current coupled ice-ocean models.

The EVP method aims to solve the VP non-linear equations without the need of a linear solver. In the one-dimensional example, the modified EVP method proposed by Lemieux et al. (2012) corresponds to the following equations:

$2 T \frac{\sigma^{p+1}-\sigma^{p}}{\Delta t_{\mathrm{e}}}+\sigma^{p}=\frac{P}{2 \Delta^{p}} \partial_{x} u^{p}$,

$\beta^{*} \frac{u^{p+1}-u^{p}}{\Delta t_{\mathrm{e}}}+m \frac{u^{p}-u_{n}}{\Delta t}=\partial_{x} \sigma^{p+1}+\tau$,

where $\beta^{*}$ is an extra parameter having the same unit as $m$. The subscripts corresponding to the global timestep are not written any more except when equal to $n$. During the sub-iterations, other variables are not updated and the forcing is kept constant. This method does not need a solver because the update of the internal stress and velocity components are decoupled and local. A crucial difference between the classical and the modified EVP method is the treatment of the physical inertial term $m \partial_{t} u$ which is discretized into $m \frac{u-u_{n}}{\Delta t}$. In the classical EVP method, it is confounded with the update of the velocity during the iterative process as the left-hand side of Eq. (21) is replaced by $m \frac{u^{p+1}-u^{p}}{\Delta t_{\mathrm{e}}}$. In consequence, the number of iterations $n_{\text {evp }}$ must be equal to $\frac{\Delta t}{\Delta t_{\mathrm{e}}}$, in order to remain consistent with the momentum equation. In the modified EVP method, the constraint on the number of iterations disappears, since the velocity update $\beta^{*} \frac{u^{p+1}-u^{p}}{\Delta t_{e}}$ and the physical inertial term $m \frac{u^{p}-u_{n}}{\Delta t}$ are treated separately. The elimination of the constraint on $n_{\text {evp }}$ allows to evaluate the error of the approximate solution during the iterative process and to stop it when a prescribed accuracy is reached.

\subsection{Reinterpretation of EVP}

In this section, we completely reinterpret EVP as an iterative solver for the system of non-linear equations obtained from the implicit time discretization of the VP problem. Applied to the one-dimensional case presented in Section 3.2, the algorithm successively executes these operations:

$\sigma^{p+1}=\sigma^{p}+\left(\hat{\sigma}^{p}-\sigma^{p}\right) \frac{1}{\alpha}$,

$u^{p+1}=u^{p}+\left(\hat{u}^{p}-u^{p}\right) \frac{1}{\beta}$,

where $\alpha$ and $\beta$ are non-dimensional parameters larger than 1 . Internal stress is firstly updated towards the target $\hat{\sigma}^{p}=P /\left(2 \Delta^{p}\right) \partial_{x} u^{p}$, then, velocity is updated towards the target $\hat{u}^{p}=u_{n}+$ $\left(\partial_{x} \sigma^{p+1}+\tau\right) \frac{\Delta t}{m}$. The proposed method exactly corresponds to the modified EVP method proposed by Lemieux et al. (2012) if the remaining two numerical parameters ( $\alpha$ and $\beta$ ) are defined by:

$\alpha=\frac{2 T}{\Delta t_{\mathrm{e}}}$

$\beta=\frac{\beta^{*}}{m} \frac{\Delta t}{\Delta t_{\mathrm{e}}}$,

where $T, \Delta t_{\mathrm{e}}$ and $\beta^{*}$ are the three numerical parameters used in Lemieux et al. (2012).

The proposed method also closely corresponds to the classical EVP method of Hunke (2001), if

$\beta=\frac{\Delta t}{\Delta t_{\mathrm{e}}}$,

and if the terms $\sigma^{p}$ and $u^{p}$ inside the parenthesis of the right hand side of Eqs. (22) and (23) are replaced by $\sigma^{p+1}$ and $u^{p+1}$. However, no significant changes in terms of convergence or stability have been found in our study when using $\sigma^{p}$ and $u^{p}$ instead of $\sigma^{p+1}$ and $u^{p+1}$.

\subsection{Convergence and stability of the method}

The iterative process corresponds to

$\sigma^{p+1}=\sigma^{p}+\left(\frac{P}{2 \Delta^{p}} \partial_{x} u^{p}-\sigma^{p}\right) \frac{1}{\alpha}$

$u^{p+1}=u^{p}+\left(\partial_{x} \sigma^{p+1}+\tau-m \frac{u^{p}-u_{n}}{\Delta t}\right) \frac{\Delta t}{m} \frac{1}{\beta}$.

To simplify the analysis and to mimic the one made by Hunke (2001), the term $m \frac{u^{p}-u_{n}}{\Delta t}$ is neglected in the following. Combining Eqs. (27) and (28) and assuming that $P$ and $\Delta$ are constant leads to:

$\left(u^{p+1}-2 u^{p}+u^{p-1}\right)+\left(u^{p}-u^{p-1}\right) \frac{1}{\alpha}-\partial_{x}^{2} u^{p} \frac{\gamma}{\alpha \beta}=\tau \frac{\Delta t}{m} \frac{1}{\alpha \beta}$,

where

$\gamma=\frac{P}{2 \Delta} \frac{\Delta t}{m}$.

Eq. (29) looks like a wave equation describing the evolution of the approximate solution during the iterative process. By dropping the non-homogeneous term and assuming solutions of the form $u^{p}(x) \sim e^{\mathrm{i}(k x-\omega)}$, one obtains the dispersion relation:

$\omega^{2}+\mathrm{i} \omega \frac{1}{\alpha}-k^{2} \frac{\gamma}{\alpha \beta}=0$,

where $\mathrm{i}=\sqrt{-1}, k$ is the wave number (with condition $k^{2} \Delta x^{2} \leqslant 1$ ) and $\omega$ is a non-dimensional complex number. For each term, the following relations have been used, respectively: 
$e^{-\mathrm{i} 2 \omega}-2 e^{-\mathrm{i} \omega}+1 \approx-\omega^{2}$,

$e^{-\mathrm{i} \omega}-1 \approx-\mathrm{i} \omega$

$e^{-\mathrm{i} \omega} \approx 1$.

The damping is controlled by the imaginary part of $\omega$, given by:

$\omega=\frac{1}{2 \alpha}\left(-i+\sqrt{\frac{1}{\xi^{2}}-1}\right)$,

where $\xi$ is the damping ratio defined by $\xi=\frac{S v}{S e}$, where $S_{\mathrm{e}}$ and $S_{\mathrm{v}}$ are two non-dimensional positive numbers defined by:

$S_{\mathrm{e}}=\sqrt{\frac{\alpha \beta}{\gamma}} \Delta x$

$S_{\mathrm{v}}=\frac{\beta}{\gamma} \frac{\Delta x^{2}}{2}$

where $\gamma$ comes from (30). The value of the damping ratio $\xi$ determines the type of damping. Oscillations occur during the approach to the solution if $\xi<1$ and the damping rate is equal to $\frac{1}{2 \alpha}$. The condition $\xi^{2}>1$ corresponds to the relation $T<\frac{e^{2} m \Delta x^{2} \Delta}{8 P}$ found by Hunke (2001).

The definitions of $S_{\mathrm{e}}$ and $S_{\mathrm{v}}$ transform Eq. (29) into the following discrete wave equation:

$\left(u^{p+1}-2 u^{p}+u^{p-1}\right) S_{\mathrm{e}}^{2}+\left(u^{p}-u^{p-1}\right) 2 S_{\mathrm{v}}-\delta_{x}^{2} u^{p}=\tau \frac{\Delta t}{m} \frac{\Delta x^{2}}{\gamma}$,

with the second order finite difference:

$\delta_{x}^{2} u^{p}=u^{p}(x+\Delta x)-2 u^{p}(x)+u^{p}(x-\Delta x)$.

The stability criterion is developed by assuming that $u^{p}=a^{p} e^{i k x}$, where $a^{p}=\lambda a^{p-1}$. By introducing a solution of this form and dropping the non-homogeneous term, Eq. (38) becomes:

$\lambda^{2}-\left(2-\frac{k^{2} \Delta x^{2}}{S_{\mathrm{e}}^{2}}-\frac{1}{\alpha}\right) \lambda+\left(1-\frac{1}{\alpha}\right)=0$.

The method is stable if $|\lambda|<1$. Using the properties $\alpha>1$ and $k^{2} \Delta x^{2} \leqslant 1$, the stability criterion is given by

$S_{\mathrm{e}}^{2}>0.5$.

This stability criterion is similar to the condition $T>\frac{P\left(1+e^{2}\right)^{2} \Delta t_{e}^{2}}{32 m \Delta x^{2} e^{2} \Delta}$ developed by Hunke (2001).

In cases where the internal stress is negligible, i.e., under ice divergence or in regions with low ice concentration, the problem reduces to:

$\beta\left(u^{p+1}-u^{p}\right)=\left(\tau \frac{\Delta t}{m}+u_{n}\right)-u^{p}$.

The residual decreases as $e^{-\frac{1}{p} p}$ and the convergence is controlled by $\frac{1}{\beta}$. The method is always stable and locally convergent.

Equivalently, for a constant strain rate, the convergence rate of the stress state is given by $\frac{1}{\alpha}$ and the distance to the correct stress state decreases as $e^{-\frac{1}{\alpha} p}$.

\subsection{Summary and details of implementation}

In summary, EVP is an ad hoc solver for the system of non-linear equations obtained from the implicit time discretization of the VP model. Convergence and stability of the method depend on the two non-dimensional numerical parameters, $\alpha$ and $\beta$. Three nondimensional positive numbers, $S_{\mathrm{e}}, S_{\mathrm{v}}$ and $\xi$, are defined and used to characterize the behaviour of the solver.
In previous implementations of EVP, numerical and physical terms were somewhat confounded, generating some confusion between the numerical method and the physical model. In the classical EVP method of Hunke (2001), the numerical parameters were interpreted as an elastic damping timescale $T$ and a subcycling timestep $\Delta t_{\mathrm{e}}$. The number of iterations $n_{\mathrm{evp}}$ had to be equal to $\frac{\Delta t}{\Delta t_{\mathrm{e}}}$. The modified EVP method of Lemieux et al. (2012) suppresses the need for a fixed number of iterations, but adds an additional parameter, $\beta^{*}$, interpreted as an artificial mass. In the revised EVP method, only two non-dimensional numerical parameters remain and the condition on the number of iterations is relaxed. The parallelization is still trivial and the computational time is unchanged.

The treatment of the stress update is slightly different from previous EVP implementations, where $2 T \partial_{t} \sigma_{1}, \frac{2 T}{e^{2}} \partial_{t} \sigma_{2}, \frac{2 T}{e^{2}} \partial_{t} \sigma_{12}$, were added to the left-hand side of Eqs. (7)-(9) to regularize the definition of the stress. The reinterpretation of EVP leads to a new regularization where $\sigma_{1}$ is not treated differently than $\sigma_{2}$ and $\sigma_{12}$. The relaxation of each stress component is then similar and is controlled by $\alpha$. To reproduce the old regularization, one can use $\alpha=\frac{2 T}{\Delta t_{\mathrm{e}}}$ for $\sigma_{1}$, and $\alpha=\frac{1}{e^{2}} \frac{2 T}{\Delta t_{\mathrm{e}}}$ for $\sigma_{2}$ and $\sigma_{12}$. Keeping the same $\alpha$ for the three equations has a surprisingly good impact on the quality of the solution as analysed in the next section.

At the first iteration $(p=1)$ of each global timestep, an initial guess for $(\boldsymbol{u}, \boldsymbol{\sigma})$ has to be defined. For the ice velocity, we take the velocity from the previous timestep. For the ice internal stress, we set $\sigma_{1}, \sigma_{2}, \sigma_{12}$ at zero at the beginning of each timestep, as it is usually done. This allows us to evaluate the quality of the stress states by the distance to the ellipse. Other initial guesses could also be used, such as the free drift velocity or a smoothed version of the previous velocity and internal stress.

The entire algorithm is detailed hereafter to simplify implementation in sea ice models. Within the iterative process, variables indexed with $p$ are updated, while variables indexed with $n$ are referred to the global timestep and stay constant. The number of iterations, $n_{\mathrm{evp}}$, can be fixed a priori or depend on a termination criterion as a function of the residual. At each solver iteration, the internal stress tensor is updated first by:

$\alpha\left(\sigma_{1}^{p+1}-\sigma_{1}^{p}\right)=\frac{P}{\left(\Delta^{p}+\Delta_{\min }\right)}\left(\dot{\epsilon}_{1}^{p}-\Delta^{p}\right)-\sigma_{1}^{p}$,

$\alpha\left(\sigma_{2}^{p+1}-\sigma_{2}^{p}\right)=\frac{P}{\left(\Delta^{p}+\Delta_{\min }\right)} \frac{\dot{\epsilon}_{2}^{p}}{e^{2}}-\sigma_{2}^{p}$,

$\alpha\left(\sigma_{12}^{p+1}-\sigma_{12}^{p}\right)=\frac{P}{\left(\Delta^{p}+\Delta_{\min }\right)} \frac{\dot{\epsilon}_{12}^{p}}{e^{2}}-\sigma_{12}^{p}$.

The internal force vector is then computed by:

$\mathbf{F}^{p+1}=\left(F_{\mathrm{u}}^{p+1}, F_{\mathrm{v}}^{p+1}\right)=\nabla \cdot \boldsymbol{\sigma}^{p+1}$

and used to update the velocity.

The updates of $u$ and $v$ are decoupled. In odd iterations, (47) is solved first with $v^{*}$ as an interpolation of $v^{p}$ on $u$-points and $u^{*}$ equal to $u^{p+1}$. Then, (48) is solved with $v^{*}=v^{p+1}$ and $u^{*}$ is $u^{p+1}$ interpolated on $v$-points. The reverse order is applied for even iterations. One therefore has:

$$
\begin{aligned}
\beta \frac{m}{\Delta t}\left(u^{p+1}-u^{p}\right)= & F_{\mathrm{u}}^{p+1}-\frac{m}{\Delta t}\left(u^{p+1}-u_{n}\right) \\
& +A\left(\tau_{a \mathrm{u}}+c_{D} \rho_{o}\left|\boldsymbol{u}_{o}-\left(u^{*}, v^{*}\right)\right|\left(u_{o}-u^{p+1}\right)\right) \\
& +m f v^{*}
\end{aligned}
$$

and

$$
\begin{gathered}
\beta \frac{m}{\Delta t}\left(v^{p+1}-v^{p}\right)=F_{\mathrm{v}}^{p+1}-\frac{m}{\Delta t}\left(v^{p+1}-v_{n}\right)+A\left(\tau_{a v}+c_{D} \rho_{o} \mid \boldsymbol{u}_{o}\right. \\
\left.-\left(u^{*}, v^{*}\right) \mid\left(v_{o}-v^{p+1}\right)\right)-m f u^{*}
\end{gathered}
$$




\section{Results and discussion}

Different simulations are performed to analyse the impacts of the numerical parameters on the quality of the solution. Comparisons between the old and new stress regularizations highlight the strong link between the generation of linear bands of high ice deformation and numerical problems.

We work in the framework of the Nucleus for European Modelling of the Ocean (NEMO) model (Madec, 2008) with the Louvain-la-Neuve sea Ice Model (LIM) discretized on a C-grid. The modifications are made to version 3.4 of NEMO-LIM (branch/ dev_r3322_NOCS09_SAS) with the LIM2 version of the sea ice model on the ORCA05 grid in an Arctic configuration (see Madec et al. (1998) and Fichefet and Maqueda (1997) for a description of LIM2). The ocean is assumed to be at rest with no elevation. The ocean stress is computed using a quadratic formula with a drag coefficient $c_{D}=5 \times 10^{-3}$. The surface wind is obtained from the 6 hourly DFS4.1 $10 \mathrm{~m}$ wind data (Brodeau et al., 2010). Surface boundary conditions (wind stress and other fluxes) are determined by the CORE bulk formulae proposed by Large and Yeager (2004). The timestep is equal to $4 \mathrm{~h}(\Delta t=14400 \mathrm{~s})$ and all simulations start the first of January 1991. To simplify the analysis, very simple initial conditions are chosen. There is no ice wherever the sea surface temperature is $2^{\circ} \mathrm{C}$ above the seawater freezing point, $3 \mathrm{~m}$ of ice and $50 \mathrm{~cm}$ of snow elsewhere. Sea surface temperatures and salinities are derived from the Levitus98 monthly climatology (Boyer et al., 1998). The initial concentration of the ice pack is equal to 1 . The ORCA05 tripolar grid has a resolution of about $15 \mathrm{~km}$ in the Canadian Arctic Archipelago and around $25 \mathrm{~km}$ in the central Arctic (Fig. 2). The domain is restricted to the ocean surface at latitudes higher than $25^{\circ}$ North. The model parameters are the following: $P^{*}=10^{4} \mathrm{~N} \mathrm{~m}^{-2}$ and $C=20$. We choose a very small value for $\Delta_{\min }\left(10^{-20} \mathrm{~s}^{-1}\right)$ in order to model nearly pure plastic flow.

An advantage of the revised EVP method is the possibility of evaluating the numerical convergence by computing the residual of the equations. Similarly to Lemieux et al. (2012), we find that the EVP method exhibits stagnation and oscillation of the residual for the standard parameters presented here above, but is able to converge to machine accuracy with a 10 times smaller ice strength

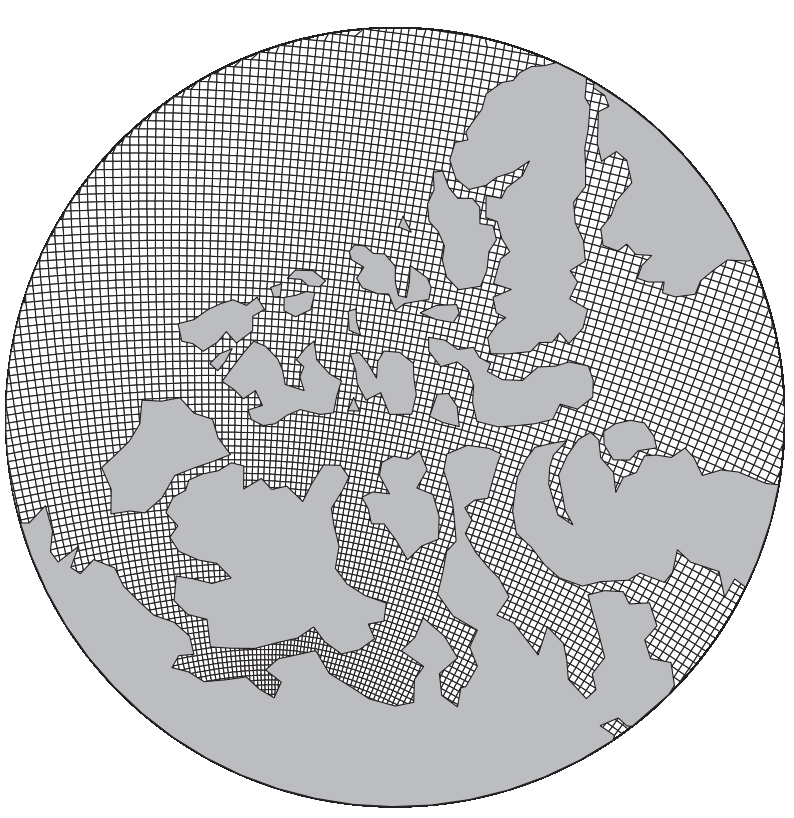

Fig. 2. ORCA05 mesh over the Canadian Arctic Archipelago (CAA). The resolution varies from $15 \mathrm{~km}$ in the CAA to about $25 \mathrm{~km}$ in the central Arctic. and $\Delta_{\min }=2 \times 10^{-9} \mathrm{~s}^{-1}$. Our corrections of the EVP method and the tuning of the numerical parameters do not help in avoiding the stagnation of the residual. This lack of numerical convergence is still an open question and is not solved in this paper. Our study focus on the effect of numerical details on unconverged solutions.

Section 4.1 concerns the choice of the values of the numerical parameters $\alpha$ and $\beta$ in order to have a correct approximation of the solution within a limited number of iterations. The effect of these parameters on stability and damping is checked by looking at three diagnostics: the evolution of the maximum increment of the velocity during the iterative process, the stress states after 300 iterations and the divergence rate field after 300 iterations in the Canadian Arctic Archipelago (CAA), which is a region where sea ice deformation is very low and where the EVP method suffers stability and underdamping problems (e.g., Hunke, 2001). The maximum increment of the velocity is computed as the largest difference in velocity components between the current and the previous iteration. An optimal set of parameters is proposed to avoid the noisy ice deformation fields in the CAA and to maintain stress states on the yield curve while avoiding oscillations during the iterative process.

In Section 4.2, two slightly different regularizations are compared, the new one where the three stress components use the same $\alpha$, and the old one that is equivalent to using $\frac{\alpha}{\rho^{2}}$ to update $\sigma_{2}$ and $\sigma_{12}$, and $\alpha$ to update $\sigma_{1}$. For each regularization, the error is evaluated a posteriori by comparing the solution with the solution obtained with the same regularization when a very large number of iterations has been used. The large remaining errors in the velocity fields with the old regularization correlates with the generation of linear bands of high deformation.

\subsection{Selection of the values of the numerical parameters}

Previous studies (e.g., Hunke, 2001) showed noisy divergence fields in nearly rigid ice regions, where $P$ is large and $\Delta$ is small. These features arise from numerical instabilities and have also been detected in Lemieux et al. (2012). To circumvent this issue, the classical approach involves reducing the subcycling timestep $\Delta t_{\mathrm{e}}$ to improve the stability criterion. However, this reduction of $\Delta t_{\mathrm{e}}$ requires a larger number of iterations, either for consistency or damping, which may be unaffordable for many applications. An alternative option that improves stability while preserving damping is to increase the value of the extra inertial term in the modified EVP method which corresponds to the $\beta$ parameter in our revised method. This idea has never been tested and will be used in this section to study the effect of numerical parameters on the quality of unresolved solutions in nearly rigid ice areas.

Here is a brief summary useful for the analysis of our results. $\alpha$ and $\beta$ are the only two numerical parameters of the solver. $\alpha$ controls the stress update and $\beta$ the velocity update. Their combination controls the stability of the method and the damping of oscillations during the solving process. $S_{\mathrm{e}}, S_{\mathrm{v}}$ and $\xi$ are three nondimensional positive numbers that can be compared from different configurations. In nearly rigid ice regions, the oscillations are underdamped as $\xi$ is much lower than 1 , and the damping rate is inversely proportional to $\alpha$. The stability criterion is given by Eq. (41).

Four simulations over a few days are performed to analyse the effects of $\alpha$ and $\beta$ (Table 2). All simulations have the same number of iterations, $n_{\mathrm{evp}}=300$, which is affordable for most applications. Only results for the first timestep are shown, but the analysis remains valid for the other timesteps. Each experiment has the same forcing and initial conditions.

To compute the diagnostics presented in Table 2, we had to evaluate the value of the non-dimensional number $\gamma$, which is a function of $\Delta t, \Delta$ and the strength-to-mass ratio of the ice. To do 
Table 2

Values of the two non-dimensional numerical parameters $\alpha$ and $\beta$ and the nondimensional number $S_{\mathrm{e}}$ for the four set of parameters used in the present study.

\begin{tabular}{llll}
\hline Name & $\alpha$ & $\beta$ & $S_{\mathrm{e}}$ \\
\hline Exp1 & 40 & 3000 & 0.87 \\
Exp2 & 98 & 122 & 0.27 \\
Exp3 & 126 & 947 & 0.86 \\
Exp4 & 40 & 300 & 0.27 \\
\hline
\end{tabular}

so, $\Delta$ is evaluated a posteriori from the simulated ice velocity field (Fig. 3 shows the logarithm of $\Delta$ after 300 iterations for Exp1). The minimum value of $\Delta$ in a cell that is not at the coast or at the ice edge is around $2 \times 10^{-9} \mathrm{~s}^{-1}$. It is a better estimate than $\Delta_{\min }$, which is in our case very small and never reached $\left(10^{-20} \mathrm{~s}^{-1}\right)$. This value leads to evaluate $\gamma=36 \times 10^{12}$ which is used to estimate $S_{\mathrm{e}}, S_{\mathrm{v}}$ and $\xi$ for the different set of parameters. Therefore, these diagnostics characterize the behaviour of the method in nearly rigid ice regions, where the oscillations are underdamped $\left(\xi^{2}<1\right)$. In regions with larger deformation rates, stability and damping conditions are relaxed since $S_{\mathrm{e}}$ and $\xi$ increase with $\sqrt{\Delta}$.

Three quantities are used to compare the four simulations. The first one is the maximum velocity update whose evolution during the iterative process indicates stability problems as shown in Fig. 4. The second quantity is the normalized stress state at iteration 300 which should be on the elliptical yield curve (Fig. 5). Note that this indicator is not completely appropriate as the stress states can be on the elliptic yield curve but not at the correct location, which is the unique point on the curve where the normal flow rule is respected. The third variable of interest is the divergence rate in the CAA (Fig. 6), given at iteration 300. This field is noisy when the solver encounters stability or underdamping problems.

Exp1 respects the stability criterion given by (41) if $\Delta>1.5 \times 10^{-9} \mathrm{~s}^{-1}$ and has the highest damping rate of the four experiments (Table 2). The maximum increment of the velocity monotonically decreases and most of the stress states are on the expected yield curve (Fig. 4 and 5). The noise in the ice divergence rate is low (Fig. 6) and only visible in Fig. 3 where the logarithm of $\Delta$ is shown.

Exp2 leads to the poorest performances. The stability criterion is only valid where $\Delta>1.5 \times 10^{-8} \mathrm{~s}^{-1}$ and the damping rate is

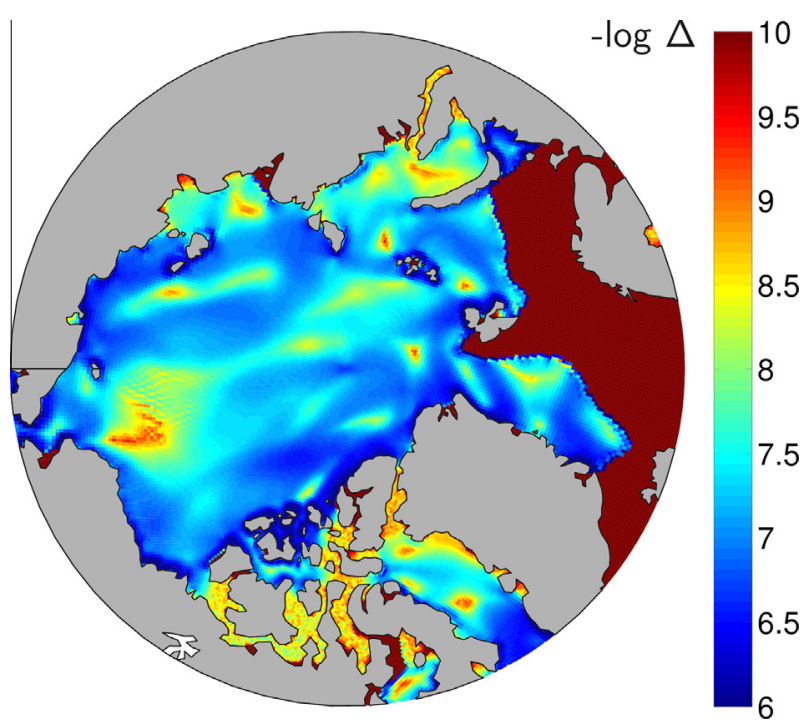

Fig. 3. Logarithm of $\Delta$ after 300 iterations for the first timestep with parameters given by Exp1. Noise in the ice deformation field is visible in narrow passages and nearly rigid ice regions but with low magnitude, around $10^{-9} \mathrm{~s}^{-1}$.

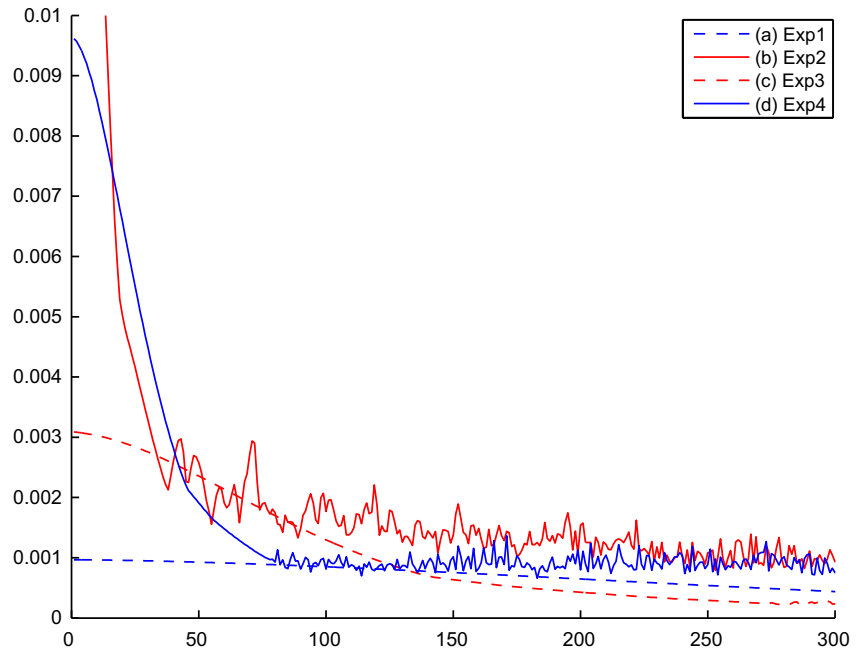

Fig. 4. Evolution of the maximum increment of the velocity $\left[\mathrm{m} \mathrm{s}^{-1}\right]$ during the first 300 iterations of the first timestep. Exp1 corresponds to the dashed blue curve Exp2 to the red curve, Exp3 to the dashed red curve and Exp4 to the blue curve.

low. Large and rapid oscillations are noticed in the evolution of the maximum increment of the velocity (red line in Fig. 4), corresponding to instabilities generated by this set of parameters. The stress states are still not on the expected ellipse after 300 iterations but are on a smaller ellipse (Fig. 5) and noise in ice deformation is observed as in Hunke (2001) (Fig. 6).

Exp3 has the same stability criterion as Exp1 but the damping rate is lower due to the higher value of $\alpha$. At first, the velocity update is higher than for Exp1 (red dashed line in Fig. 4), but it decreases more rapidly and starts to slightly oscillate in the last 20 iterations. The distance of the stress state to the ellipse is still large after 300 iterations (Fig. 5). Noise in ice deformation is observed in the CAA but to a lesser extent and with a smaller magnitude than in Exp2 and Exp4 (Fig. 6).

In Exp4, the damping property is restored to the detriment of the stability criteria $S_{\mathrm{e}}$, which drops down to the same value as in Exp2. Exp4 has stress states near the ellipse thanks to the smaller value of $\alpha$ (Fig. 5) but experiences an oscillatory behaviour similar to that seen in Exp2 (blue line in Fig. 4). Deformation fields remain noisy in the CAA (Fig. 6).

To summarize, Exp2 and Exp4 do not respect the stability criterion where $\Delta$ is low and exhibit large oscillations in the maximum increment of the velocity and stagnation of its value around $0.1 \mathrm{~cm} \mathrm{~s}^{-1}$. Exp1 and Exp3 respect the stability criterion where $\Delta>1.5 \times 10^{-9} \mathrm{~s}^{-1}$, but Exp3 exhibits weak oscillations in the maximum increment of the velocity and little noise in ice deformation in the CAA, which are associated with stress states far from the ellipse and caused by the large value of $\alpha$. In conclusion, the noise in the CAA can have two origins: either insufficient damping of the oscillations or instabilities of the method. Both problems are addressed in Exp1 which has a well-reproduced yield curve and limited noise in the ice deformation field in nearly rigid ice regions.

Because of the poor convergence and stability of the EVP method, the solutions obtained after a limited number of iterations are strongly influenced by the numerical parameters and may exhibit noise in the deformation fields and poorly resolved stress states. Our analysis presents a new way to reduce instabilities and oscillations. By playing with the value of $\beta$ one can preclude a noisy ice deformation field while maintaining stress states near the ellipse without any additional computational cost. In the following section, another impact of numerical details is explored. 

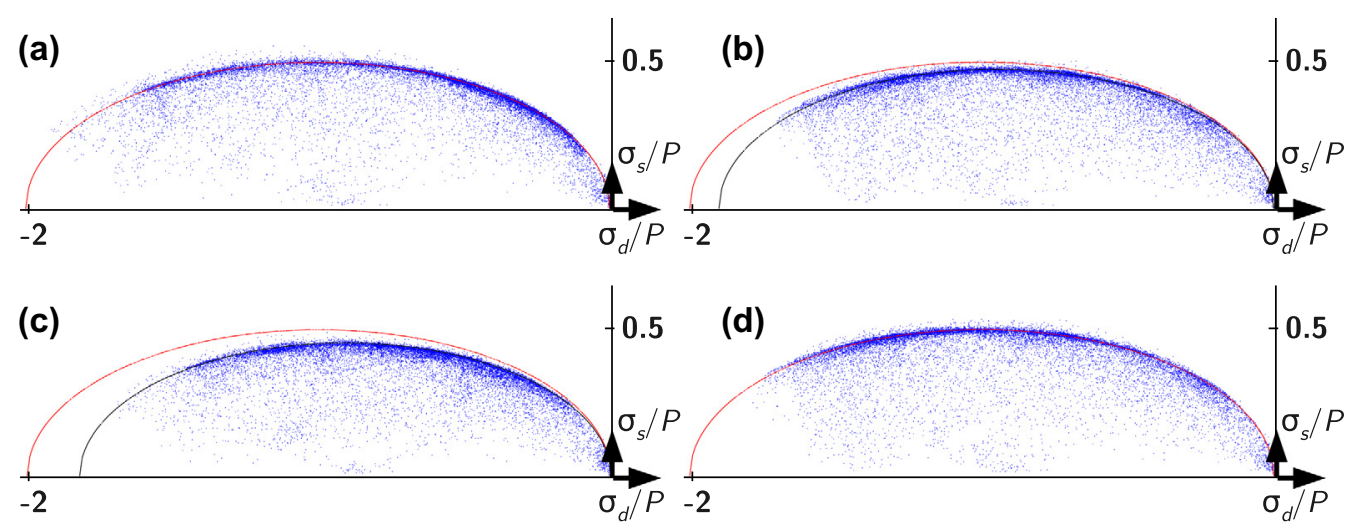

Fig. 5. Internal ice stress normalized by the ice strength $P$ for the entire domain (except the cells near the coast) after 300 iterations for the first timestep of experiments Exp1 (a), Exp2 (b), Exp3 (c) and Exp4 (d). The red curve is the reference ellipse. The black curve in the panel (b) and (c) corresponds to the reference ellipse scaled by 0.95 and 0.91 , respectively.
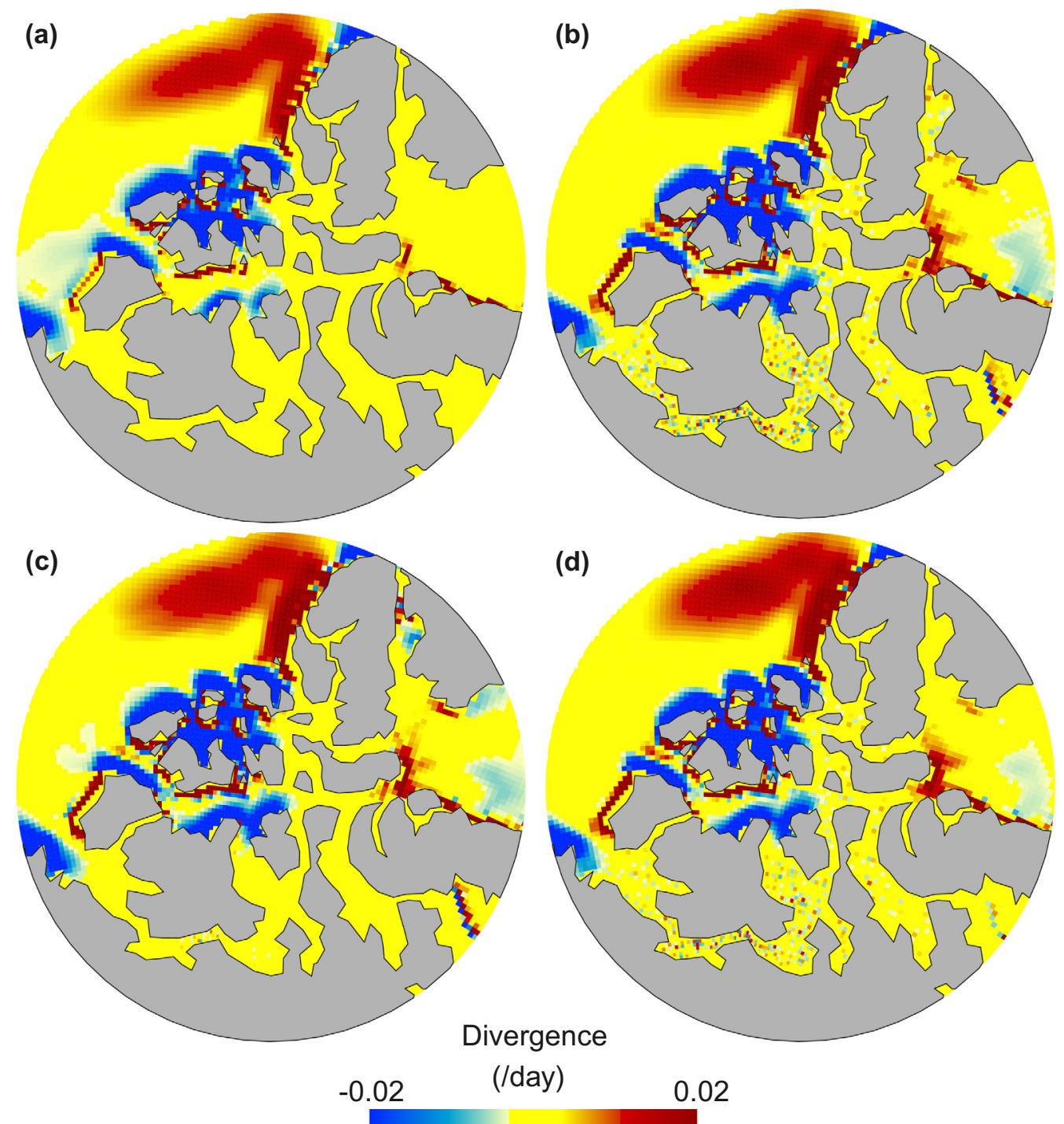

Fig. 6. Sea ice divergence rate within the Canadian Arctic Archipelago after 300 iterations for the first timestep of experiments Exp1 (a), Exp2 (b), Exp3 (c) and Exp4 (d). $0.02 \mathrm{day}^{-1}$ is approximately equal to $2 \times 10^{-7} \mathrm{~s}^{-1}$.

\subsection{Comparison of the old and new regularizations}

To mimic the original regularization of EVP used in previous studies (Hunke, 2001; Hunke and Dukowicz, 2002; Bouillon et al., 2009; Lemieux et al., 2012), the value of $\alpha$ is divided by $e^{2}$ for updating $\sigma_{2}$ and $\sigma_{12}$. This small difference between the two regularization does not change the equations for which the solver is called but has a large impact on the quality of the solution. The 
effect of using the old regularization is clearly visible when looking at the stress state after 300 iterations obtained with the set of parameters having the poorest performances (Exp2). The solution obtained with the old regularization (see Fig. 7) does not have the expected aspect ratio, indicating faster convergence in the shear stress direction than in the compressive stress direction. The stress states obtained with the new regularization shown on

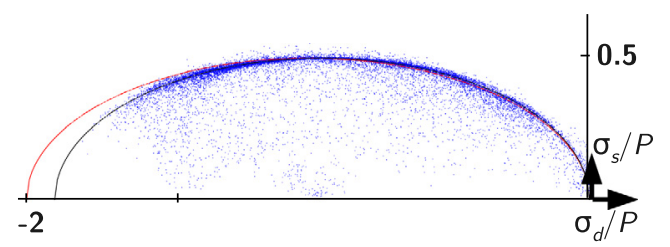

Fig. 7. Internal ice stress normalized by the ice strength $P$ for all the domain (except the cells near the coast) after 300 iterations for the first timestep of the same experiment as Exp2 but with the old regularization instead of the new one. The red curve is the reference ellipse which has an aspect ratio equal to $e$. The black ellipse is contracted in the compressive dimension by a factor 0.95 .
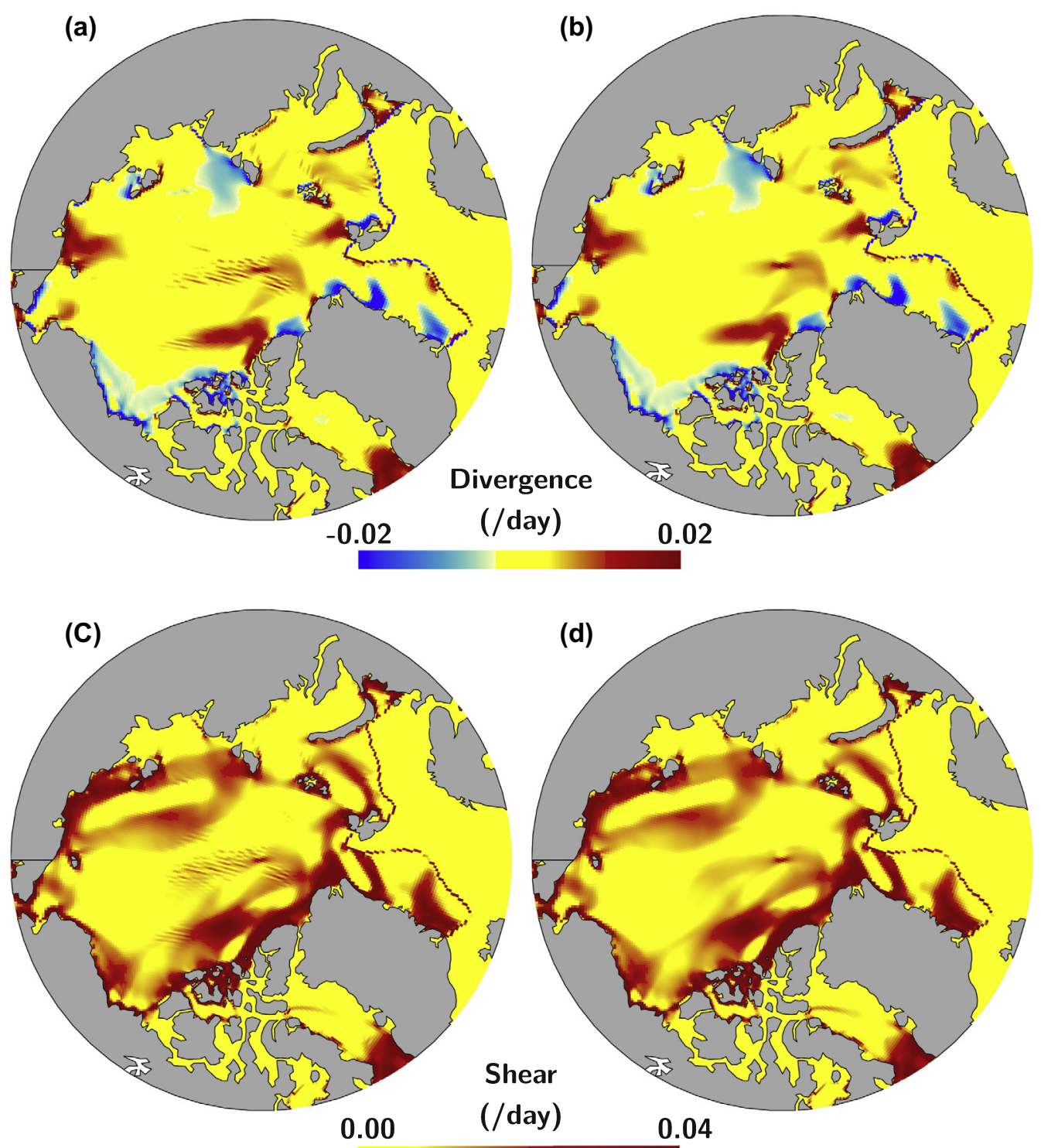

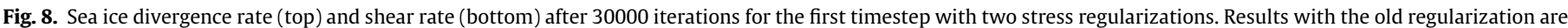

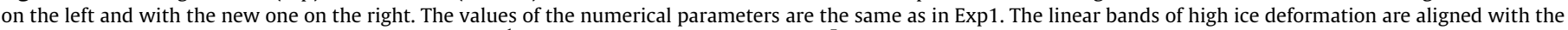
grid in both directions and are one cell wide. $0.02 \mathrm{day}^{-1}$ is approximately equal to $2 \times 10^{-7} \mathrm{~s}^{-1}$.
Fig. 5(b) are not on the expected yield curve either but are located on an ellipse having the correct aspect ratio thanks to the identical treatment of the stress components. With more iterations, both solutions have stress states on the correct yield curve but still lead to different deformation and velocity fields.

Results with the two regularizations are now compared with the same parameters, initial conditions and external forcing as in Exp1. The analysis presented here has been verified for all sets of parameters, with no noticeable change. The two regularizations are compared in terms of simulated deformation fields and remaining errors in the velocity fields.

Fig. 8 displays the ice divergence and shear rates obtained after 30000 iterations with the two versions of the solver. The maximum velocity update at iteration 30000 is about $0.4 \times 10^{-5} \mathrm{~ms}^{-1}$ with the two versions. The old regularization produces linear bands of high ice deformation which are not present with the new regularization. High positive values for the divergence are observed in those bands which are aligned with the grid in both directions and are one cell wide. In the rest of the domain, deformation and velocity fields are similar between the two versions of the code. 
With both versions, no noise is observed in the ice deformation fields in the CAA, and the stress states are well located on the ellipse (not shown).

The error at iteration 300 (or 3000) is measured by computing the differences in velocity between the approximate solutions at iteration 300 (or 3000) and 30000. The error after 300 iterations is of the same order of magnitude for both versions (Fig. 9). The maximum error in the velocity components is $0.13 \mathrm{~cm} \mathrm{~s}^{-1}$ for the old regularization and $0.08 \mathrm{~cm} \mathrm{~s}^{-1}$ for the new one. After 3000 iterations, the maximum error is reduced by a factor of 2 for the old regularization and by 40 for the new one $\left(0.06\right.$ and $0.002 \mathrm{~cm} \mathrm{~s}^{-1}$, respectively) (Fig. 9). In both cases, the average error decreases, but locally, the error remains significant in large parts of the domain when the old regularization is used. The linear bands of high ice deformation are correlated with large up-and-down variations in the profile of the velocity field in both directions (up to $0.06 \mathrm{~cm} \mathrm{~s}^{-1}$ ) (not shown). Such structures in the velocity field are not observed with the new regularization except in narrow passages (e.g., Nares Strait), but with a much lower magnitude of $0.0005 \mathrm{~cm} \mathrm{~s}^{-1}$.
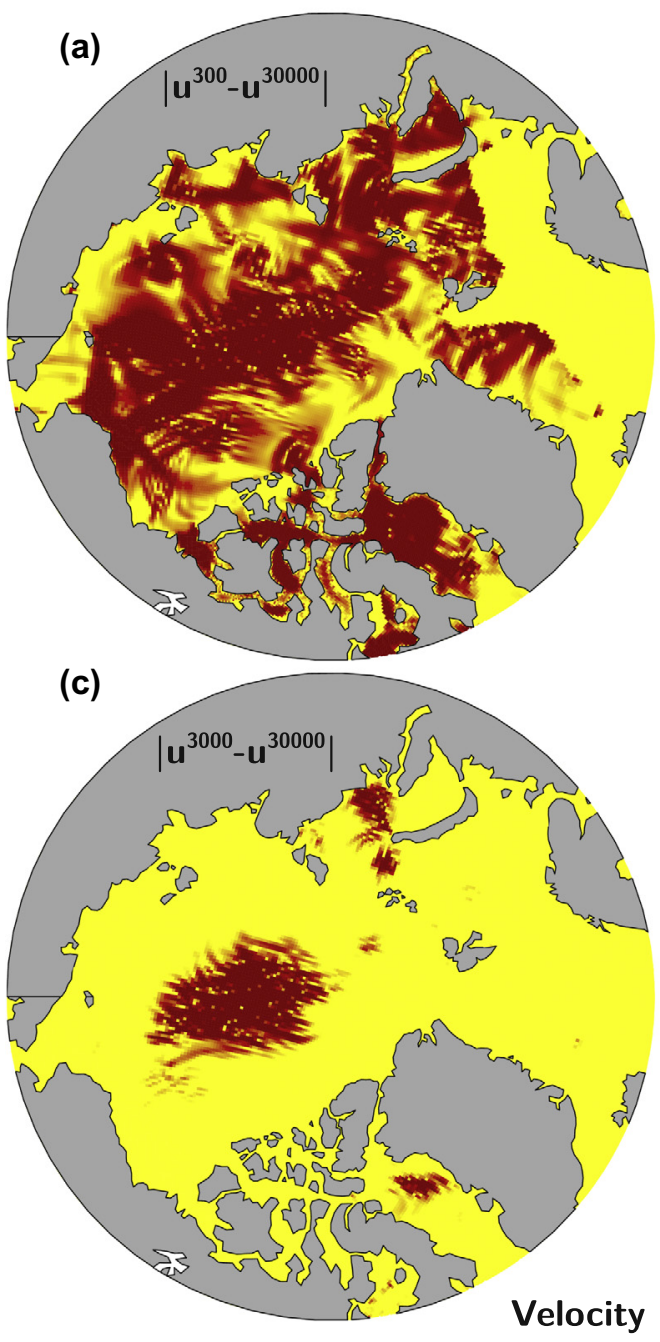

(d)
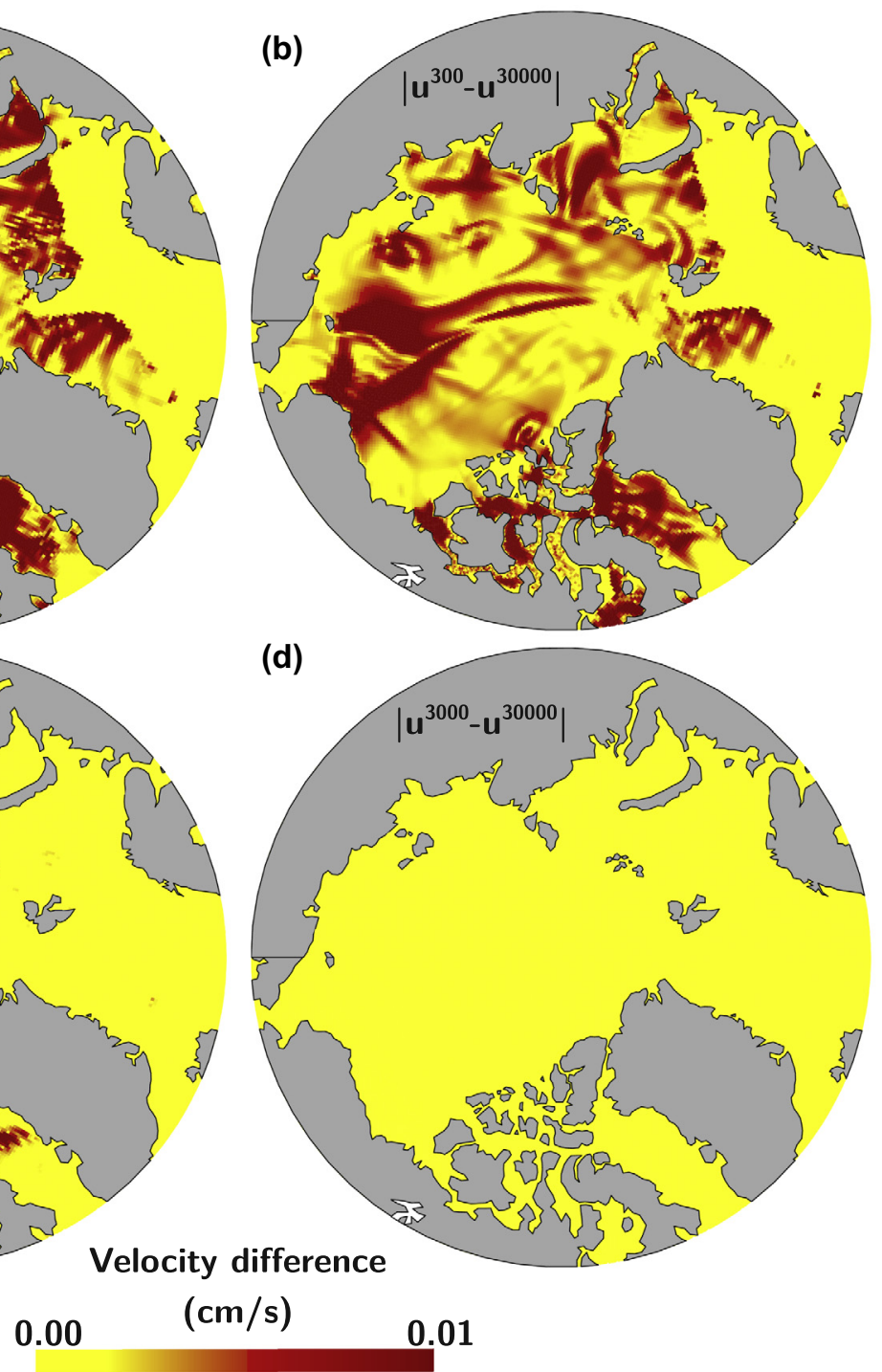
bands aligned with the grid. deformation stripes.

Our explanation of the problematic behaviour of the old regularization is that the different treatment of the stress components excites oscillations and causes the production of high deformation

Other studies present deformation fields with parallel bands of high deformation aligned with the numerical grid. These stripes in the divergence and shear fields are similar to the patterns produced by our simulation with the old regularization (see Fig. 8 (a) and (c)). Lemieux et al. (2012) compared solutions given by the classical EVP method to a fully converged solution obtained with a Picard scheme and a tight non-linear convergence criterion. The EVP solver after about 2000 iterations captures the general pattern of ice deformation but produces additional high deformation features. Some of these features are aligned with the grid and are organized into stripes (see the divergence rate field on their Fig. 8f). The deformation fields obtained by Maslowski and Lipscomb (2003) with EVP at a $9 \mathrm{~km}$ resolution also exhibit high

The similarities between these studies and the simulation with the old regularization presented here may help in clarifying the origin of some of the linear bands of high ice deformation. We

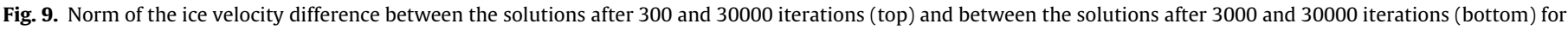

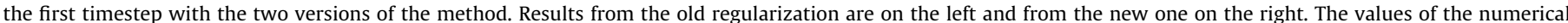

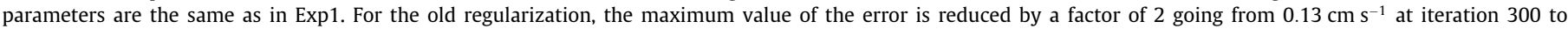
$0.06 \mathrm{~cm} \mathrm{~s}^{-1}$ at iteration 30000. For the new one, it is reduced by a factor of 40 going from $0.08 \mathrm{~cm} \mathrm{~s}^{-1}$ at iteration 300 to $0.002 \mathrm{~cm} \mathrm{~s} \mathrm{~s}^{-1}$ at iteration 30000 . 
suggest that the cause of the generation of these high deformation stripes can be the poor numerical behaviour of the methods used to solve the non-linear VP problem. Their appearance is characterized by large remaining errors in the velocity field and the alignment with the grid is further evidence of poor numerical behaviour.

\section{Conclusions}

In this paper, we reinterpret the EVP model as an iterative solver. This allows us to define a clear distinction between the numerical and physical parameters and to remove the constraint on the number of iterations. Similarly to previous studies, we find that the EVP method has poor numerical convergence. In consequence numerical details may have significant impact on the approximate solution.

The new numerical parameters gives clear criteria to control the stability of the method and the damping of oscillations during the iterative process. We also propose a set of parameters that avoid the noisy deformation fields in nearly rigid ice regions as in the Canadian Arctic Archipelago (CAA).

By analysing the stress states during the iterative process, we find that the relaxation of the compressive stress component is lower than the relaxation of the shear stress components, with the classical EVP formulation proposed by Hunke and Dukowicz (1997). We propose a new regularization of the internal stress to correct this discrepancy. Comparison between the old and new regularizations illustrates the strong impact of numerical details on solutions obtained with the EVP method even after a large number of iterations. The old regularization produces linear bands of high deformation aligned with the grid that may be confounded with real linear kinematic features but are in fact the results of a numerical artefact. Their appearance correlates with the persistence of significant errors in the velocity field in large parts of the domain even after many iterations. All the implementations based on this regularization may exhibit the same behaviour.

In previous studies, similar features were obtained numerically (Wang and Wang, 2009; Maslowski and Lipscomb, 2003) and compared to linear kinematic features observed by Kwok (2006). Our analysis indicates that in some cases such features could arise from numerical artefacts and correlate with large remaining errors in the velocity field.

\section{Acknowledgements}

With support from IsENES european project (http://is.enes.org) and from MyOcean2 european project (http://www.myocean.eu).

\section{References}

Bouillon, S., Maqueda, M.A.M., Legat, V., Fichefet, T., 2009. An elastic-viscousplastic sea ice model formulated on Arakawa B and C grids. Ocean Modelling 27, 174-184.

Boyer, T., Levitus, S., Antonov, J., Conkright, M., O’Brien, T., Stephens, C., 1998. World ocean database 1998. vol. 1: Temperature of the atlantic ocean. Tech. rep., NOAA Atlas NESDIS 27, US Government Printing Office, Washington, DC, pp. 166

Brodeau, L., Barnier, B., Treguier, A.-M., Penduff, T., Gulev, S., 2010. An era40-based atmospheric forcing for global ocean circulation models. Ocean Modelling 31 (3), 88-104.

Connolley, W.M., Gregory, J.M., Hunke, E., McLaren, A.J., 2004. On the consistent scaling of terms in the sea-ice dynamics equation. Journal of Physical Oceanography $34,1776-1780$.

Coon, M., Kwok, R., Levy, G., Pruis, M., Schreyer, H., Sulsky, D., 2007. Arctic ice dynamics joint experiment (AIDJEX) assumptions revisited and found inadequate. Journal of Geophysical Research 112, C11S90.
Fichefet, T., Goosse, H., Maqueda, M.A.M., 1998. Ocean Modeling and Parameterization. On the large-scale modeling of sea ice-ocean, interactions, vol. C516. Kluwer Academic Publisher, Dordrecht, pp. 399-422.

Fichefet, T., Maqueda, M.A.M., 1997. Sensitivity of a global sea ice model to the treatment of ice thermodynamics and dynamics. Journal of Geophysical Research 102, 12609-12646.

Girard, L., Bouillon, S., Weiss, J., Amitrano, D., Fichefet, T., Legat, V., 2011. A new modelling framework for sea ice mechanics based on elasto-brittle rheology. Annals of Glaciology 52 (57), 123-132.

Girard, L., Weiss, J., Molines, J., Barnier, B., Bouillon, S., 2009. Evaluation of highresolution sea ice models on the basis of statistical and scaling properties of arctic sea ice drift and deformation. Journal of Geophysical Research (C8).

Hibler, W.D., 1979. A dynamic thermodynamic sea ice model. Journal of Physical Oceanography 9, 817-846.

Hibler, W.D., Schulson, E., 2000. On modelling the anisotropic failure and flow of flawed sea ice. Journal of Geophysical Research 105, 17105-17120.

Hunke, E., Dukowicz, J., 2003. The sea ice momentum equation in the free drift regime. Tech. Rep., LA-UR-03-2219, Los Alamos National Laboratory.

Hunke, E.C., 2001. Viscous-plastic sea ice dynamics with the EVP model: linearization issues. Journal of Computational Physics 170, 18-38.

Hunke, E.C., Dukowicz, J.K., 2002. The elastic-viscous-plastic sea ice dynamics model in general orthogonal curvilinear coordinates on a sphere-incorporation of metric terms. Monthly Weather Review 130, 1848-1865.

Hunke, E.C., Dukowicz, J.K., 1997. An elastic-viscous-plastic model for sea ice dynamics. Journal of Physical Oceanography 27, 1849-1867.

Hunke, E.C., Lipscomb, W.H., 2008. Cice: the los alamos sea ice model documentation and software user's manual. Tech. Rep., T-3 Fluid Dynamics Group, Los Alamos National Laboratory, Los Alamos NM 87545.

Hunke, E.C., Zhang, Y., 1999. A comparison of sea ice dynamics models at high resolution. Monthly Weather Review 127, 396-408.

Kwok, R., 2006. Contrasts in sea ice deformation and production in the arctic seasonal and perennial ice zones. Journal of Geophysical Research 111 (C11), C11S22.

Kwok, R., Hunke, E.C., Maslowski, W., Menemenlis, D., Zhang, J., 2008. Variability of sea ice simulations assessed with RGPS kinematics. Journal of Geophysical Research 113, C11012.

Large, W., Yeager, S., 2004. Diurnal to decadal global forcing for ocean and sea-ice models: the data sets and flux climatologies. NCAR Tech. Note TN-460+ STR.

Lemieux, J.-F, Knoll, D., Tremblay, B., Holland, D., Losch, M., 2012. A comparison of the jacobian-free Newton-Krylov method and the EVP model for solving the sea ice momentum equation with a viscous-plastic formulation: a serial algorithm study. Journal of Computational Physics 231 (17), 5926-5944.

Lemieux, J.-F., Tremblay, B., 2009. Numerical convergence of viscous-plastic sea ice models. Journal of Geophysical Research 114, C05009.

Lemieux, J.-F., Tremblay, B., Sedláček, J., Tupper, P., Thomas, S., Huard, D., Auclair, J.P., 2010. Improving the numerical convergence of viscous-plastic sea ice models with the Jacobian-free Newton-Krylov method. Journal of Computational Physics 229, 2840-2852.

Losch, M., Danilov, S., 2012. On solving the momentum equations of dynamic sea ice models with implicit solvers and the elastic-viscous-plastic technique. Ocean Modelling 41, 42-52.

Losch, M., Menemenlis, D., Campin, J.-M., Heimbach, P., Hill, C., 2010. On the formulation of sea-ice models. Part 1: Effects of different solver implementations and parameterizations. Ocean Modelling 33 (1-2), 129-144.

Madec, G., May 2008. NEMO Ocean engine. Pôle de modélisation de l'Institut PierreSimon Laplace.

Madec, G., Delecluse, P., Imbard, M., Lévy, C., 1998. OPA 8.1 Ocean genera circulation model reference manual. Note du Pôle de modélisation, Tech. Rep. XX, Institut Pierre Simon Laplace (IPSL) pp. 91.

Martin, T., Gerdes, R., 2007. Sea ice drift variability in arctic ocean model intercomparison project models and observations. Journal of Geophysical Research 112.

Maslowski, W., Lipscomb, W., 2003. High resolution simulations of arctic sea ice, 1979-1993. Polar Research 22 (1), 67-74

Schreyer, H.L., Sulsky, D.L., Munday, L.B., Coon, M.D., Kwok, R., 2006. Elasticdecohesive constitutive model for sea ice. Journal of Geophysical Research 111, C11S26.

Wang, K., Wang, C., 2009. Modeling linear kinematic features in pack ice. Journal of Geophysical Research 114 (C12), C12011.

Wilchinsky, A.V., Feltham, D.L., 2004. A continuum anisotropic model of sea-ice dynamics. Royal Society 460, 2105-2140.

Zhang, J., Hibler, W.D., 1997. On an efficient numerical method for modelling sea ice dynamics. Journal of Geophysical Research 102, 8691-8702.

Zhang, J., Rothrock, D., 2000. Modeling arctic sea ice with an efficient plastic solution. Journal of Geophysical Research 105 (C2), 3325-3338.

Zhang, J., Rothrock, D.A., 2005. Effect of sea ice rheology in numerical investigations of climate. Journal of Geophysical Research 110, C08014. 